\title{
A Remark on Regularity of Solutions to Wave Equations
}

\author{
Naoya SAEKI and Takeshi WADA \\ Kumamoto University \\ (Communicated by H. Nawa)
}

Abstract. We prove a generalization of the Strichartz estimate for the inhomogeneous wave equation $\square u(t, x)=f(t, x)$ in the space-time $\boldsymbol{R}^{1+n}$. We estimate the solution in vector-valued homogeneous Besov spaces $\dot{B}_{q, 2}^{\theta}\left(\boldsymbol{R} ; \dot{B}_{r, 2}^{\sigma}\left(\boldsymbol{R}^{n}\right)\right)$. Such an estimate shows the time differentiability of the solution of fractional order.

\section{Introduction}

In this paper, we study the following Cauchy problem for the wave equation:

$$
\begin{aligned}
& \square u(t, x)=f(t, x), \\
& u(0, x)=u_{0}(x), \quad \partial_{t} u(0, x)=u_{1}(x) .
\end{aligned}
$$

Here, $(t, x) \in \boldsymbol{R}^{1+n}$ with $n \geq 2$ and $\square=\partial_{t}^{2}-\Delta$ is the usual d'Alembertian.

It is well-known that the solution to the wave equation satisfies the inequality called "Strichartz estimate", which shows space-time integrability of the solution $[6,10,14,7]$. Similar estimates are also known for various kinds of wave and dispersive equations, e.g., the Klein-Gordon [2, 4, 10, 14, 13], the Schrödinger [5, 17, 14], and the Airy (linear part of the K-dV) [8] equations, and have played important roles in the analysis of related nonlinear problems.

We begin with the definition of function spaces. For a function $f$ defined on $\boldsymbol{R}^{n}$, we define the Fourier transform of $f$ by $\hat{f}(\xi)=(\mathscr{F} f)(\xi)=\int_{\boldsymbol{R}^{n}} e^{-i x \xi} f(x) d x$ and the inverse Fourier transform of $\hat{f}$ by $\left(\mathscr{F}^{-1} \hat{f}\right)(x)=\int_{\boldsymbol{R}^{n}} e^{i x \xi} \hat{f}(\xi) d \xi$ respectively, where $d \xi=(2 \pi)^{-n} d \xi$. Let $\omega=(-\Delta)^{1 / 2}=\mathscr{F}^{-1}|\xi| \mathscr{F}$. For $s \in \boldsymbol{R}$ and $1 \leq r \leq \infty$, the homogeneous Sobolev space $\dot{H}_{r}^{s}=\dot{H}_{r}^{s}\left(\boldsymbol{R}^{n}\right)$ is defined by $\dot{H}_{r}^{s}=\omega^{-s} L^{r}$, where $L^{r}=L^{r}\left(\boldsymbol{R}^{n}\right)$ denotes the usual Lebesgue space. If $r=2$, we simply write $\dot{H}^{s}=\dot{H}_{2}^{s}$. To define the homogeneous Besov space $\dot{B}_{r}^{s}=\dot{B}_{r, 2}^{s}\left(\boldsymbol{R}^{n}\right)$, we need the Littlewood-Paley decomposition. Let $\eta \in C_{0}^{\infty}(\boldsymbol{R})$ be a nonnegative even function satisfying supp $\eta \subset\{\tau ; 1 / 2 \leq|\tau| \leq 2\}$ and

Received May 29, 2014

Mathematics Subject Classification: 35L05

Key words and phrases: Wave equations, Strichartz estimates, Besov spaces 
$\sum_{j=-\infty}^{\infty} \eta\left(\tau / 2^{j}\right)=1$ for $\tau \neq 0$. For any $j \in \boldsymbol{Z}$, we put $\varphi_{j}(x)=\int_{\boldsymbol{R}^{n}} e^{i x \xi} \eta\left(|\xi| / 2^{j}\right) d \xi$. The homogeneous Besov space is then defined by

$$
\dot{B}_{r}^{s}=\left\{u \in \mathscr{S}^{\prime}\left(\boldsymbol{R}^{n}\right) ;\left\|u ; \dot{B}_{r}^{s}\right\| \equiv\left\{\sum_{j=-\infty}^{\infty} 2^{2 s j}\left\|\varphi_{j} \underset{(x)}{*} u ; L^{r}\right\|^{2}\right\}^{1 / 2}<\infty\right\} .
$$

Precisely, we identify two tempered distributions if their difference is a polynomial since $\left\|u ; \dot{B}_{r}^{s}\right\|=0$ if and only if $u$ is a polynomial. By the Plancherel theorem, we see that $\dot{H}^{s}=$ $\dot{B}_{2}^{s}$. On the other hand, $\dot{H}_{r}^{s} \subset \dot{B}_{r}^{s}$ if $1<r<2$ and $\dot{H}_{r}^{s} \supset \dot{B}_{r}^{s}$ if $2<r<\infty$. We refer the reader to $[1,15]$ for further details about these spaces.

Similarly we define spaces of vector-valued functions. For a Banach space $E$ and $1 \leq$ $q \leq \infty$, let $L^{q}(E)=L^{q}(\boldsymbol{R} ; E)$. We put $\phi_{j}(t)=\int_{\boldsymbol{R}} e^{i t \tau} \eta\left(\tau / 2^{j}\right) d \tau$ for any $j \in \boldsymbol{Z}$. For any $\theta \in \boldsymbol{R}$ and $1 \leq q \leq \infty$, we define the $E$-valued Besov space $\dot{B}_{q}^{\theta}(E)=\dot{B}_{q, 2}^{\theta}(\boldsymbol{R} ; E)$ by

$$
\dot{B}_{q}^{\theta}(E)=\left\{u \in \mathscr{S}^{\prime}(\boldsymbol{R} ; E) ;\left\|u ; \dot{B}_{q}^{\theta}(E)\right\| \equiv\left\{\sum_{j=-\infty}^{\infty} 2^{2 \theta j}\left\|\phi_{j} \underset{(t)}{*} u ; L^{q}(E)\right\|^{2}\right\}^{1 / 2}<\infty\right\} .
$$

For vector-valued Besov spaces, we refer the reader to [12].

Now we precisely state the Strichartz estimate. Following the notation in [6], we introduce the symbols

$$
\beta(r)=\frac{n+1}{2}\left(\frac{1}{2}-\frac{1}{r}\right), \quad \gamma(r)=(n-1)\left(\frac{1}{2}-\frac{1}{r}\right), \quad \delta(r)=n\left(\frac{1}{2}-\frac{1}{r}\right),
$$

and $\bar{r}=r /(r-1)$ for $1 \leq r \leq \infty$. The solution to (1)-(2) satisfies the following inequality:

$$
\left\|u ; L^{q}\left(\dot{B}_{r}^{s-\beta}\right)\right\|+\left\|\partial_{t} u ; L^{q}\left(\dot{B}_{r}^{s-\beta-1}\right)\right\| \lesssim\left\|\left(u_{0}, u_{1}\right) ; \dot{H}^{s} \times \dot{H}^{s-1}\right\|+\left\|f ; L^{\bar{q}}\left(\dot{B}_{\bar{r}}^{s+\beta-1}\right)\right\|,
$$

where $s \in \boldsymbol{R}, 0 \leq 2 / q=\gamma(r) \leq 1$ with $(n, q, r) \neq(3,2, \infty)$, and $\beta=\beta(r)$. For the proof, see $[6,10,14]$ when $q \neq 2$ and [7] when $q=2$ respectively.

On the one hand, by the inequality (3) we can estimate fractional order spatial-derivatives of $u$; on the other hand, this inequality contains only the first order time-derivative of $u$. Since the wave equation is second order both in $t$ and $x$, it is natural to expect that the solution should have the same regularity in both variables. Indeed, Brenner [2] has pointed out that such an estimate holds for the homogeneous Klein-Gordon equation. For the homogeneous wave equation, namely (1)-(2) with $f=0$, the corresponding estimate $\left\|u ; \dot{B}_{q}^{\theta}\left(\dot{B}_{r}^{s-\beta}\right)\right\| \lesssim$ $\left\|\left(u_{0}, u_{1}\right) ; \dot{H}^{s+\theta} \times \dot{H}^{s+\theta-1}\right\|$ easily follows from (3) as in the proof of Theorem 1 below. We can further expect that such an estimate holds for the inhomogeneous equation if the inhomogeneous term has appropriate regularity. Pecher [11] proved such an estimate for the inhomogeneous Schrödinger equation, and Uchizono-Wada [16] slightly improved his estimate. Their methods are also applicable for the wave equation, and the corresponding 
inequality is the following:

$$
\begin{aligned}
& \left\|u ; \dot{B}_{q}^{\theta}\left(\dot{B}_{r}^{s-\beta}\right)\right\|+\left\|\partial_{t} u ; \dot{B}_{q}^{\theta}\left(\dot{B}_{r}^{s-\beta-1}\right)\right\| \lesssim\left\|\left(u_{0}, u_{1}\right) ; \dot{H}^{s+\theta} \times \dot{H}^{s+\theta-1}\right\| \\
& \quad+\left\|f ; \dot{B}_{\bar{q}}^{\theta}\left(\dot{B}_{\bar{r}}^{s+\beta-1}\right)\right\|+\sum_{ \pm}\left\|f ; L^{q_{1}(\theta \pm \varepsilon)}\left(\dot{B}_{r_{1}(\theta \pm \varepsilon)}^{s+\beta-1}\right)\right\| .
\end{aligned}
$$

Here, $\varepsilon>0$ is a sufficiently small number, and $q_{1}(\theta), r_{1}(\theta)$ are defined by

$$
1 / q_{1}(\theta)=(2 \theta-1) / q+1-\theta, \quad \delta\left(r_{1}(\theta)\right)=1 / q_{1}(\theta)-1+\theta-\beta .
$$

However, the inequality (4) is not optimal from the scaling point of view. To see this, we consider the scale change

$$
(u, f) \mapsto\left(u_{\lambda}, f_{\lambda}\right) \equiv\left(\lambda^{n / 2-s} u(\lambda t, \lambda x), \lambda^{n / 2-s+2} f(\lambda t, \lambda x)\right) .
$$

We can easily check that both (1) and (3) are invariant under this scaling. On the other hand, (4) is not scale-invariant, and therefore is not optimal.

In the present paper, we aim at improving Pecher's estimate and obtaining a scaleinvariant estimate for the fractional time-derivative of the solution to (1)-(2). In order to state our theorem, we introduce the space $\dot{Z}_{q, r}^{s}$, which is the completion of $\dot{\mathscr{S}}\left(\boldsymbol{R}^{1+n}\right)=\{u \in$ $\mathscr{S}\left(\boldsymbol{R}^{1+n}\right) ; \int_{\boldsymbol{R}^{n}} x^{\alpha} u(t, x) d x=0$ for any $\left.\alpha \in \boldsymbol{Z}_{+}^{n}\right\}$ by the norm

$$
\left\|u ; \dot{Z}_{q, r}^{s}\right\|=\left\{\sum_{j=-\infty}^{\infty} 2^{2 s j}\left\|\varphi_{j} \underset{(x)}{*} u ; L^{q}\left(L^{r}\right)\right\|^{2}\right\}^{1 / 2} \sim\left\{\sum_{j=-\infty}^{\infty}\left\|\varphi_{j} \underset{(x)}{*} u ; L^{q}\left(\dot{B}_{r}^{s}\right)\right\|^{2}\right\}^{1 / 2} .
$$

The main theorem in this paper is the following:

THEOREM 1. Let $s \in \boldsymbol{R}$ and $0<\theta<1$. Let $1 \leq q, r, q_{1}, r_{1} \leq \infty$ satisfy $0 \leq 2 / q=$ $\gamma(r) \leq 1$ with $(n, q, r) \neq(3,2, \infty), r_{1} \leq 2$ and $1 / q_{1}-\delta\left(r_{1}\right)=1-\theta+\beta$ with $\beta=\beta(r)$. Then for any $\left(u_{0}, u_{1}\right) \in \dot{H}^{s+\theta} \times \dot{H}^{s+\theta-1}$ and $f \in \dot{B}_{\bar{q}}^{\theta}\left(\dot{B}_{\bar{r}}^{s+\beta-1}\right) \cap \dot{Z}_{q_{1}, r_{1}}^{s+\beta-1}$, the solution to (1)-(2) satisfies the estimate

$$
\begin{aligned}
& \left\|u ; \dot{B}_{q}^{\theta}\left(\dot{B}_{r}^{s-\beta}\right)\right\|+\left\|\partial_{t} u ; \dot{B}_{q}^{\theta}\left(\dot{B}_{r}^{s-\beta-1}\right)\right\| \\
& \quad \lesssim\left\|\left(u_{0}, u_{1}\right) ; \dot{H}^{s+\theta} \times \dot{H}^{s+\theta-1}\right\|+\left\|f ; \dot{B}_{\bar{q}}^{\theta}\left(\dot{B}_{\bar{r}}^{s+\beta-1}\right) \cap \dot{Z}_{q_{1}, r_{1}}^{s+\beta-1}\right\| .
\end{aligned}
$$

REMARK 1. (i) We can easily check that both sides of (6) is invariant under the transform (5) with $s$ replaced by $s+\theta$.

(ii) Minkowski's inequality shows $\dot{Z}_{q_{1}, r_{1}}^{s+\beta-1} \supset L^{q_{1}}\left(\dot{B}_{r_{1}}^{s+\beta-1}\right)$ if $q_{1} \leq 2$, and $\dot{Z}_{q_{1}, r_{1}}^{s+\beta-1} \subset$ $L^{q_{1}}\left(\dot{B}_{r_{1}}^{s+\beta-1}\right)$ if $q_{1} \geq 2$.

(iii) If $\theta \leq 1 / 2$, then $q_{1}(\theta) \leq 2$. Therefore, putting $\left(q_{1}, r_{1}\right)=\left(q_{1}(\theta), r_{1}(\theta)\right)$, we have $\dot{Z}_{q_{1}, r_{1}}^{s+\beta-1} \supset L^{q_{1}}\left(\dot{B}_{r_{1}}^{s+\beta-1}\right) \supset \bigcap_{ \pm} L^{q_{1}(\theta \pm \varepsilon)}\left(\dot{B}_{r_{1}(\theta \pm \varepsilon)}^{s+\beta-1}\right)$. Therefore (4) follows from (6). On the other hand, (4) does not follow from (6) when $\theta>1 / 2$, although (6) is a scale-invariant refinement of (4). Even in this case, a slight modification of the proof of Theorem 1 gives a simpler, alternative proof of (4) (see the remark at the end of §2). 
(iv) The space $\dot{Z}_{1,2}^{s}$ (precisely the inhomogeneous counterpart of this space) was introduced by Chemin and Lerner [3] for the analysis of the Navier-Stokes equations.

(v) Similar estimate also holds for the Schrödinger equation [9].

\section{Proof of Theorem}

We put $v_{ \pm}=\partial_{t} \omega^{-1} u \pm i u$ and rewrite (1)-(2) to the following first-order system:

$$
\begin{aligned}
& \partial_{t} v_{ \pm} \mp i \omega v_{ \pm}=\omega^{-1} f, \\
& v_{ \pm}(0)=v_{0, \pm}=\omega^{-1} u_{1} \pm i u_{0} .
\end{aligned}
$$

Furthermore, by the propagator $U_{ \pm}(t)=\exp ( \pm i t \omega)$, we convert (7)-(8) to the integral equations

$$
v_{ \pm}(t)=U_{ \pm}(t) v_{0, \pm}+\int_{0}^{t} U_{ \pm}\left(t-t^{\prime}\right) \omega^{-1} f\left(t^{\prime}\right) d t^{\prime} .
$$

Since $\dot{B}_{r}^{s}=\omega^{-s} \dot{B}_{r}^{0}$, without loss of generality we may assume $s=0$. Therefore the inequality (3) is clearly equivalent to

$$
\left\|v_{ \pm} ; L^{q}\left(\dot{B}_{r}^{-\beta}\right)\right\| \lesssim\left\|v_{0, \pm} ; L^{2}\right\|+\left\|f ; L^{\bar{q}}\left(\dot{B}_{\bar{r}}^{\beta-1}\right)\right\|,
$$

and Theorem 1 follows from the proposition below:

Proposition 1. Let $0<\theta<1$. Let $1 \leq q, r, q_{1}, r_{1} \leq \infty$ satisfy $0 \leq 2 / q=\gamma(r) \leq$ 1 with $(n, q, r) \neq(3,2, \infty), r_{1} \leq 2$ and $1 / q_{1}-\delta\left(r_{1}\right)=1-\theta+\beta$ with $\beta=\beta(r)$. Then for any $v_{0, \pm} \in \dot{H}^{\theta}$ and $f \in \dot{B}_{\bar{q}}^{\theta}\left(\dot{B}_{\bar{r}}^{\beta-1}\right) \cap \dot{Z}_{q_{1}, r_{1}}^{\beta-1}$, the solution to (7)-(8) satisfies the estimate

$$
\left\|v_{ \pm} ; \dot{B}_{q}^{\theta}\left(\dot{B}_{r}^{-\beta}\right)\right\| \lesssim\left\|v_{0, \pm} ; \dot{H}^{\theta}\right\|+\left\|f ; \dot{B}_{\bar{q}}^{\theta}\left(\dot{B}_{\bar{r}}^{\beta-1}\right) \cap \dot{Z}_{q_{1}, r_{1}}^{\beta-1}\right\| .
$$

ProOF. We only prove the estimate for $v_{+}$, so that we omit the subscript " \pm ". We can estimate the homogeneous and the inhomogeneous terms separately, and the homogeneous estimate is quite easy. Indeed, the Fourier transform shows $\phi_{j} *_{(t)} U(t) v_{0}=U(t) \varphi_{j} *_{(x)} v_{0}$, and hence we obtain by (10)

$$
\begin{aligned}
\left\|U(t) v_{0} ; \dot{B}_{q}^{\theta}\left(\dot{B}_{r}^{-\beta}\right)\right\|^{2} & =\sum_{j=-\infty}^{\infty} 2^{2 \theta j}\left\|U(t) \varphi_{j} \underset{(x)}{*} v_{0} ; L^{q}\left(\dot{B}_{r}^{-\beta}\right)\right\|^{2} \\
& \lesssim \sum_{j=-\infty}^{\infty} 2^{2 \theta j}\left\|\varphi_{j} \underset{(x)}{*} v_{0} ; L^{2}\right\|^{2} \sim\left\|v_{0} ; \dot{H}^{\theta}\right\|^{2} .
\end{aligned}
$$

We proceed to the inhomogeneous estimate. In what follows we assume $v_{0}=0$, so that

$$
v(t)=\int_{0}^{t} U\left(t-t^{\prime}\right) \omega^{-1} f\left(t^{\prime}\right) d t^{\prime} .
$$

Then we see by the Fourier transform 


$$
\begin{aligned}
\hat{v}(t, \xi) & =\int_{0}^{t} e^{i\left(t-t^{\prime}\right)|\xi|}|\xi|^{-1} \hat{f}\left(t^{\prime}, \xi\right) d t^{\prime}=\int_{0}^{t} e^{i\left(t-t^{\prime}\right)|\xi|}|\xi|^{-1} d t^{\prime} \int_{-\infty}^{\infty} e^{i t^{\prime} \tau} \tilde{f}(\tau, \xi) d \tau \\
& =\int_{-\infty}^{\infty} \frac{e^{i t \tau}-e^{i t|\xi|}}{i|\xi|(\tau-|\xi|)} \tilde{f}(\tau, \xi) d \tau .
\end{aligned}
$$

Here, $\tilde{f}(\tau, \xi)$ denotes the Fourier transform of $f$ in the space-time. Therefore, $v=v_{1}-v_{2}$ with

$$
\begin{aligned}
& \hat{v}_{1}(t, \xi)=\text { p.v. }-\int_{-\infty}^{\infty} \frac{e^{i t \tau}}{i|\xi|(\tau-|\xi|)} \tilde{f}(\tau, \xi) d \tau \\
& \hat{v}_{2}(t, \xi)=e^{i t|\xi|} \text { p.v. }-\int_{-\infty}^{\infty} \frac{1}{i|\xi|(\tau-|\xi|)} \tilde{f}(\tau, \xi) d \tau \equiv e^{i t|\xi|} \hat{\psi}_{0}(\xi)
\end{aligned}
$$

By the residue theorem,

$$
\text { p.v. }-\int_{-\infty}^{\infty} \frac{e^{i t \tau}}{i(\tau-|\xi|)} d \tau=\frac{1}{2} \operatorname{sign}(t) e^{i t|\xi|},
$$

and hence we obtain

$$
v(t)=\frac{1}{2} \int_{-\infty}^{\infty} \operatorname{sign}\left(t-t^{\prime}\right) U\left(t-t^{\prime}\right) \omega^{-1} f\left(t^{\prime}\right) d t^{\prime}-U(t) \psi_{0} .
$$

Therefore, using the formula $\phi_{j} *_{(t)} U(t)=U(t) \varphi_{j} *_{(x)}$ and the inequality (10), we can show

$$
\begin{aligned}
\left\|v ; \dot{B}_{q}^{\theta}\left(\dot{B}_{r}^{-\beta}\right)\right\|^{2} & =\sum_{j=-\infty}^{\infty} 2^{2 \theta j} \| \phi_{j} \underset{(t)}{* v ; L^{q}\left(\dot{B}_{r}^{-\beta}\right) \|^{2}} \\
& \lesssim \sum_{j=-\infty}^{\infty} 2^{2 \theta j}\left\|\phi_{j} \underset{(t)}{*} f ; L^{\bar{q}}\left(\dot{B}_{\bar{r}}^{\beta-1}\right)\right\|^{2}+\sum_{j=-\infty}^{\infty} 2^{2 \theta j}\left\|\varphi_{j} \underset{(x)}{*} \psi_{0} ; L^{2}\right\|^{2} .
\end{aligned}
$$

The first term of the right-hand side is $\left\|f ; \dot{B}_{\bar{q}}^{\theta}\left(\dot{B}_{\bar{r}}^{\beta-1}\right)\right\|^{2}$. To estimate the second term, we further decompose

$$
\begin{aligned}
\hat{\varphi}_{j}(\xi) \hat{\psi}_{0}(\xi) & =\text { p.v. }-\int_{-\infty}^{\infty} \frac{\hat{\varphi}_{j}(\xi) \hat{\chi}_{j}(\tau)}{i|\xi|(\tau-|\xi|)} \tilde{f}(\tau, \xi) d \tau+\int_{-\infty}^{\infty} \frac{\hat{\varphi}_{j}(\xi)\left(1-\hat{\chi}_{j}(\tau)\right)}{i|\xi|(\tau-|\xi|)} \tilde{f}(\tau, \xi) d \tau \\
& \equiv \hat{\psi}_{1 j}(\xi)+\hat{\psi}_{2 j}(\xi),
\end{aligned}
$$

where $\hat{\chi}_{j}(\tau)=\sum_{k=j-2}^{j+2} \hat{\phi}_{k}(\tau)$. Similarly as above, the formula (13) yields

$$
\psi_{1 j}=-\frac{1}{2} \int_{-\infty}^{\infty} \operatorname{sign}(t) U(-t) \varphi_{j} \underset{(x)}{*} \chi_{j} \underset{(t)}{*} \omega^{-1} f(t) d t .
$$


We estimate $\psi_{1 j}$ by a duality argument. To that end, we take an arbitrary function $w \in L^{2}$. Since $U(t)$ is a unitary operator in $L^{2}$, from the inequality (10) we see

$$
\begin{aligned}
\left|\left(\psi_{1 j}, w\right)_{L^{2}}\right| & \leq \frac{1}{2} \int_{-\infty}^{\infty}\left|\left(\varphi_{j} \underset{(x)}{*} \chi_{j} \underset{(t)}{*} \omega^{-1} f(t), U(t) w\right)_{L^{2}}\right| d t \\
& \leq \frac{1}{2}\left\|\chi_{j} \underset{(t)}{*} \omega^{-1} f ; L^{\bar{q}}\left(\dot{B}_{\bar{r}}^{\beta}\right)\right\|\left\|U(t) w ; L^{q}\left(\dot{B}_{r}^{-\beta}\right)\right\| \\
& \lesssim\left\|\chi_{j} \underset{(t)}{*} \omega^{-1} f ; L^{\bar{q}}\left(\dot{B}_{\bar{r}}^{\beta}\right)\right\|\left\|w ; L^{2}\right\| .
\end{aligned}
$$

Since $w$ is arbitrary, we obtain $\left\|\psi_{1 j} ; L^{2}\right\| \lesssim\left\|\chi_{j} *_{(t)} f ; L^{\bar{q}}\left(\dot{B}_{\bar{r}}^{\beta-1}\right)\right\|$, which yields

$$
\sum_{j=-\infty}^{\infty} 2^{2 \theta j}\left\|\psi_{1 j} ; L^{2}\right\|^{2} \lesssim\left\|f ; \dot{B}_{\bar{q}}^{\theta}\left(\dot{B}_{\bar{r}}^{\beta-1}\right)\right\|^{2}
$$

We next consider $\psi_{2 j}$. We put

$$
K(t, x)=\iint_{\boldsymbol{R}^{1+n}} e^{i t \tau+i x \xi} \frac{\hat{\varphi}_{0}(|\xi|)\left(1-\hat{\chi}_{0}(\tau)\right)}{i(\tau-|\xi|)} d \tau d \xi .
$$

On the support of the integrand, we have $|\tau-| \xi|| \geq 1 / 4$. Therefore, the integration by parts shows that $(1+|t|+|x|)^{m} K(t, x) \in L^{\infty}\left(\boldsymbol{R}^{1+n}\right)$ for any nonnegative integer $m$. We also introduce the rescaled function $K_{j}(t, x)=2^{n j} K\left(2^{j} t, 2^{j} x\right)$. Then

$$
\psi_{2 j}(x)=\iint_{\boldsymbol{R}^{1+n}} K_{j}\left(-t, x-x^{\prime}\right) \sum_{k=j-2}^{j+2} \varphi_{k} \underset{(x)}{*} \omega^{-1} f\left(t, x^{\prime}\right) d t d x^{\prime} .
$$

For $q_{1}, r_{1}$ in the assumption of the proposition, we can choose $1 \leq q_{0}, r_{0} \leq \infty$ such that $1 / q_{0}+1 / q_{1}=1 / r_{0}+1 / r_{1}-1 / 2=1$. Applying the Hölder and the Young inequalities, we see

$$
\begin{aligned}
\left\|\psi_{2 j} ; L^{2}\right\| & \leq\left\|K_{j} ; L^{q_{0}}\left(L^{r_{0}}\right)\right\| \sum_{k=j-2}^{j+2}\left\|\varphi_{k} \underset{(x)}{*} \omega^{-1} f ; L^{q_{1}}\left(L^{r_{1}}\right)\right\| \\
& =2^{\left(n-1 / q_{0}-n / r_{0}\right) j}\left\|K ; L^{q_{0}}\left(L^{r_{0}}\right)\right\| \sum_{k=j-2}^{j+2}\left\|\varphi_{k} \underset{(x)}{*} \omega^{-1} f ; L^{q_{1}}\left(L^{r_{1}}\right)\right\| \\
& \sim 2^{(\beta-\theta) j} \sum_{k=j-2}^{j+2} 2^{-k}\left\|\varphi_{k} \underset{(x)}{*} f ; L^{q_{1}}\left(L^{r_{1}}\right)\right\|,
\end{aligned}
$$

since $n-1 / q_{0}-n / r_{0}=1 / q_{1}-\delta\left(r_{1}\right)-1=\beta-\theta$. Therefore, we obtain

$$
\sum_{j=-\infty}^{\infty} 2^{2 \theta j}\left\|\psi_{2 j} ; L^{2}\right\|^{2} \lesssim \sum_{j=-\infty}^{\infty} 2^{2(\beta-1) j}\left\|\varphi_{j} \underset{(x)}{*} f ; L^{q_{1}}\left(L^{r_{1}}\right)\right\|^{2}=\left\|f ; \dot{Z}_{q_{1}, r_{1}}^{\beta-1}\right\|^{2} .
$$


Collecting these estimates, we can prove the proposition.

REMARK 2. Let $\varepsilon>0$ be a sufficiently small number, and let $1 / q_{1, \pm}-\delta\left(r_{1, \pm}\right)=$ $1-(\theta \pm \varepsilon)+\beta$. Then we see

$$
\begin{aligned}
\sum_{j=-\infty}^{\infty} 2^{2 \theta j}\left\|\psi_{2 j} ; L^{2}\right\|^{2} \lesssim & \sum_{j=-\infty}^{-1} 2^{2(\beta-1+\varepsilon) j}\left\|\varphi_{j} \underset{(x)}{*} f ; L^{q_{1,-}}\left(L^{r_{1,-}}\right)\right\|^{2} \\
& +\sum_{j=0}^{\infty} 2^{2(\beta-1-\varepsilon) j}\left\|\varphi_{j} \underset{(x)}{*} f ; L^{q_{1,+}}\left(L^{r_{1,+}}\right)\right\|^{2} \\
\lesssim & \left\|f ; L^{q_{1,-}}\left(\dot{B}_{r_{1,-}}^{\beta-1}\right)\right\|^{2}+\| f ; L^{q_{1,+}}\left(\dot{B}_{r_{1,+}-1}^{\beta-1} \|^{2} .\right.
\end{aligned}
$$

Therefore we obtain

$$
\left\|v_{ \pm} ; \dot{B}_{q}^{\theta}\left(\dot{B}_{r}^{-\beta}\right)\right\| \lesssim\left\|v_{0, \pm} ; \dot{H}^{\theta}\right\|+\left\|f ; \dot{B}_{\bar{q}}^{\theta}\left(\dot{B}_{\bar{r}}^{\beta-1}\right)\right\|+\sum_{ \pm}\left\|f ; L^{q_{1, \pm}}\left(\dot{B}_{r_{1, \pm}}^{\beta-1}\right)\right\| .
$$

ACKNOWLEDGment. The authors would like to thank the editor and the anonymous reviewers for their helpful comments.

\section{References}

[ 1 ] J. Bergh and J. LÖFSTRÖM, Interpolation Spaces. An Introduction, Springer, Berlin-Heidelberg-New York, Springer (1976).

[ 2 ] P. BRENNER, On space-time means and everywhere defined scattering operators for nonlinear Klein-Gordon equations, Math. Z. 186 (1984), 383-391.

[ 3 ] J.-Y. CHEMIN and N. LERNER, Flot de champs de vecteurs non lipschitziens et équations de Navier-Stokes, J. Differential Equations 121 (1995), 314-328.

[4] J. Ginibre and G. Velo, Time decay of finite energy solutions of the nonlinear Klein-Gordon and Schrödinger equations, Ann. Inst. H. Poincaré Phys. Théor. 43 (1985), 399-442.

[ 5 ] J. Ginibre and G. Velo, The global Cauchy problem for the nonlinear Schrödinger equation revisited, Ann. Inst. H. Poincaré Anal. Non Linéaire 2 (1985), 309-327.

[6] J. Ginibre and G. Velo, Generalized Strichartz inequalities for the wave equation, J. Funct. Anal. 133 (1995), 50-68.

[ 7 ] M. KeEL and T. TAO, Endpoint Strichartz estimates, Amer. J. Math. 120 (1998), 955-980.

[ 8 ] C. E. Kenig, G. Ponce and L. VegA, On the (generalized) Korteweg-de Vries equation, Duke Math. J. 59 (1989), 585-610.

[9] M. NAKAmURA and T. WADA, Modified Strichartz estimates with an application to the critical nonlinear Schrödinger equation, Nonlinear Anal., to appear.

[10] H. PeChER, Nonlinear small data scattering for the wave and Klein-Gordon equation, Math. Z. 185 (1984), 261-270.

[11] H. Pecher, Solutions of semilinear Schrödinger equations in $H^{s}$, Ann. Inst. H. Poincaré, Phys. Theor. 67 (1997), 259-296.

[12] H.-J. SchmeISSER, Vector-valued Sobolev and Besov spaces, Seminar analysis of the Karl-WeierstraßInstitute of Mathematics 1985/86 (Berlin, 1985/86), Teubner-Texte Math. 96, Teubner, Leipzig (1987), $4-44$. 
[13] I. SEgAL, Space-time decay for solutions of wave equations, Adv. Math. 22 (1976), 305-311.

[14] R. STRICHARTZ, Restrictions of Fourier transforms to quadratic surfaces and decay of solutions of wave equations, Duke Math. J. 44 (1977), 705-714.

[15] H. TRIEBEL, Interpolation Theory, Function spaces, Differential Operators, North-Holland, Amsterdam-New York-Oxford (1978).

[16] H. UChIZONO and T. WADA, On well-posedness for nonlinear Schrödinger equations with power nonlinearity in fractional order Sobolev spaces, J. Math. Anal. Appl. 395 (2012), 56-62.

[17] K. Yajima, Existence of solutions for Schrödinger evolution equations, Comm. Math. Phys. 110 (1987), $415-426$

Present Addresses:

NAOYA SAEKI

GRADUATE SCHOOL OF SCIENCE AND TECHNOLOGY,

KUMAMOTO UNIVERSITY,

KUMAMOTO 860-8555, JAPAN.

TAKESHI WADA

DEPARTMENT OF MATHEMATICS,

SHIMANE UNIVERSITY,

MATSUE 690-8504, JAPAN.

e-mail:wada@riko.shimane-u.ac.jp 